\title{
Target Localization in a Multi-Static Passive Radar System through Convex Optimization
}

Batu K. Chalise, Yimin D. Zhang, Moeness G. Amin, and Braham Himed

\section{Abstract}

We propose efficient target localization methods for a passive radar system using time-of-arrival (TOA) information of the signals received from multiple illuminators, where the position of the receiver is subject to random errors. Since the maximum likelihood (ML) formulation of this target localization problem is a non-convex optimization problem, semi-definite relaxation (SDR)-based optimization methods in general do not provide satisfactory performance. As a result, approximated ML optimization problems are proposed and solved with SDR plus bisection methods. For the case without position error, it is shown that the relaxation guarantees a rank-one solution. The optimization problem for the case with position error involves only

\footnotetext{
${ }^{2}$ B. K. Chalise is currently with Arraycomm LLC, San Jose, CA 95110, USA. He was with Villanova University, Villanova, PA, 19085, USA.

整 Y. D. Zhang, and M. G. Amin are with the Wireless Communications and Positioning Laboratory, Villanova University, Villanova, PA 19085, USA.

B. Himed is with the Air Force Research Laboratory, AFRL/RYMD, Dayton, OH 45433, USA.

The work of B. K. Chalise, Y. D. Zhang, and M. G. Amin was supported in part by a subcontract with Dynetics, Inc. for research sponsored by the Air Force Research Laboratory (AFRL) under Contract FA8650-08-D-1303, and by a subcontract with Defense Engineering Corporation for research sponsored by the AFRL under Contract FA8650-12D-1376.

The corresponding author's email address is yimin.zhang@villanova.edu.
} 
a relaxation of a scalar quadratic term. Simulation results show that the proposed algorithms outperform existing methods and provide root mean-square error performance very close to the Cramer-Rao lower bound.

Keywords: Radar signal processing, passive radar, target localization, convex optimization, semi-definite relaxation.

\section{Introduction}

In recent years, multi-static passive radar (MPR) systems, which utilize multiple broadcast signals as sources of opportunity, have attracted significant interests due to their low cost, covertness, and availability of rich illuminator sources $[1,2,3,4]$. Compared to conventional active radar systems which typically operate in a monostatic mode and emit stronger signals with a wide signal bandwidth, MPR systems use broadcast signals which in general are very weak and have an extremely narrow bandwidth. These features make it difficult to exploit a MPR system for accurate target position estimation. In addition, MPR receivers may often be implemented on aerial or ground moving vehicles. In this case, the radar platform may only have inaccurate knowledge about its own instantaneous position. This uncertainty is caused by the accuracy limitation of the positioning system as well as multipath propagations.

Target localization is an important task that has received extensive attention in various applications, such as wireless communications, sensor networks, urban canyon, and through-the-wall radar systems [5, 6, 7, 8]. Specifically, multi-lateration techniques utilize the range information observed at 
multiple positions, which are distributed over a region, to uniquely localize a target. Depending on the applications, range information can be obtained using time-of-arrival (TOA), time-delay-of-arrival (TDOA), and received signal strength indicator (RSSI). On the other hand, the observation positions may be achieved using fixed receivers, or synthesized using a single moving platform. In the latter case, the receiver positions are subject to inaccuracy.

In all these applications, maximum likelihood (ML) estimation is considered as a powerful method of estimating the targets' location, which in general is a non-convex optimization problem. When the measurement noise is sufficiently small, the ML estimation problem may be solved using linearized least squares (LLS) estimation methods [5, 7]. The key steps of the LLS estimation methods are linearizing the objective function using Taylor's series expansion at some initial guess of target position and updating it with the least squares (LS) solution in an iterative approach. Like in many iterative optimization techniques for non-convex problems, however, the accuracy of the LLS estimator highly depends on the initial guess of the target's location. This has motivated researchers to consider more efficient designs. One such approach is the semi-definite relaxation (SDR) technique $[6,9,10,11]$, which converts a non-convex optimization problem into a convex one by relaxing certain rank constraints. It is worth noting that SDR-based approaches outperform computationally efficient two-step weighted least squares method proposed in [12], especially when the noise level is high and the sensor positions are not perfectly known.

The accuracy (or tightness) of SDR techniques, however, is problem spe- 
cific, as shown in [13] for the TOA based optimization problems. For example, in optimization problems based on TDOA [9] and TOA [10], where an unknown time instant of the source's signal transmission is also optimized, SDR relaxations may not be tight and, thus, the penalty function approach is introduced. This is true also for robust designs where sensor positions are subject to certain random errors $[9,10]$. In this context, neglecting the second-order noise terms [12], the authors in [14] proposed to use an approximate ML function in the SDR-based source localization problem.

In this paper, we pursue approximated ML estimation approach in developing efficient target localization algorithms in a MPR system using TOA information of signals received from multiple illuminators and the target. As discussed above, the range resolution is poor because of the narrow signal bandwidth and weak signal levels, and the receiver position is subject to inaccurate knowledge of its own position. Therefore, an optimization problem is also formulated for the case where the receiver position is subject to estimation error. The underlying optimization problems are still non-convex, but can be reformulated as convex problems using SDR and solved in conjunction with the bisection method. When no position error is present, the SDR provides a rank-one solution. With position estimation error, the corresponding optimization problem involves only a relaxation of a quadratic scalar term.

The target localization problem and optimization technique described in this paper differs from existing literature in a number of ways. In contrast to the optimization problems in [6] and [13], where the objective function 
is solely a function of monostatic range, the objective function in our case involves bistatic range, which makes accurate target position estimation much more challenging. Further, unlike [9] and [10], where SDR of several variables and a penalty function approach are employed, our approach involves SDR of only one variable, though in conjunction with the bisection approach. For these reasons, the proposed method outperforms methods in $[9,10]$ and does not require refinement through local optimization. Moreover, although we employ ML approximation approach, the effect of the approximation renders our optimization problem to be different from [14] due to different system models. As a result, a new optimization method that solves relaxed SDR problem in conjunction with bisection method is proposed for both cases where receiver position is perfectly and imperfectly known.

The rest of the paper is organized as follows. The system model of the MPR system is described in Section 2, whereas the proposed optimization methods for target localization are presented in Section 3.2. The computational complexity of the proposed method is compared with the approach of [9] in Section 4. Numerical results are provided in Section 5 and conclusions are drawn in Section 6.

Notations: Upper (lower) bold face letters will be used for matrices (vectors); $(\cdot)^{T}, \mathbf{I}_{n},\|\cdot\|, \operatorname{tr}(\cdot), \mathbf{A} \succeq 0, \operatorname{diag}(\cdot)$ denote transpose, $n \times n$ identity matrix, Euclidean norm, matrix trace operator, positive semi-definiteness of A, and diagonal matrix, respectively. 


\section{System Model}

We consider a standard MPR system with $M$ illuminators of opportunity, which can be broadcast stations for digital-video broadcasting - terrestrial (DVB-T) [15] or base stations for global system for mobile communications (GSM) [16]. Because the deployment scenario of these broadcast and base stations is publicly available, their numbers and locations are considered to be precisely known. A narrow-band multi-frequency (NBMF) transmission is considered, where the illuminators use well separated carrier frequencies $f_{i}, i=1, \ldots, M$, and the bandwidths of their transmitted waveforms are much smaller than $f_{i}, \forall i$. The radar receiver observes the direct signals from all $M$ illuminators and the reflected signal from a single target. The target is assumed to be stationary ${ }^{1}$. By virtue of NBMF transmission, separation of the different signals based on carrier frequencies (or equivalently illuminators) is feasible after demodulation and filtering [17]. It is worthwhile to note that this feature is in contrast to a multiple-input multiple-output (MIMO) radar system where transmitters use a same carrier frequency but coordinate to form orthogonal waveforms [18], [19].

The TOA of the direct signal from the $i$ th illuminator at the receiver, where $1 \leq i \leq M$, is given by

$$
\tau_{\mathrm{d}, i}=\frac{1}{c}\left\|\mathbf{t}_{i}-\tilde{\mathbf{r}}\right\|
$$

where $c$ is the speed of light, $\mathbf{t}_{i}$ and $\tilde{\mathbf{r}}$ are column vectors of length $n$ that

\footnotetext{
${ }^{1}$ Algorithms for multiple and moving targets will be reported elsewhere.
} 
represent, respectively, the coordinates of the $i$ th illuminator and the receiver. Depending on applications, $n$ is 2 for a two-dimensional coordinate system and 3 for a three-dimension coordinate system. Note that $\left\{\mathbf{t}_{i}, \forall i\right\}$ are assumed to be stationary and precisely known. The TOA of the target reflected signal corresponding to the $i$ th illuminator is given by

$$
\tau_{\mathrm{b}, i}=\frac{1}{c}\left\{\left\|\mathbf{t}_{i}-\mathbf{p}\right\|+\|\tilde{\mathbf{r}}-\mathbf{p}\|\right\}
$$

where $\mathbf{p}$ is the $n \times 1$ vector representing the location information of the target. Because the passive radar exploits non-cooperative illuminators, it does not know the exact timing of the signal emission at each illuminator ${ }^{2}$. Rather, the radar receiver reconstructs the direct path signal with enough accuracy by applying demodulation and forward error correction technique [20]. The estimated direct path is used to correlate the reflected signal from the targets to estimate the TDOA between these two paths, given by $\tau_{\mathrm{b}, i}-\tau_{\mathrm{d}, i}[21]$. As a result, the effective TDOA between $\tau_{\mathrm{d}, i}$ and $\tau_{\mathrm{b}, i}$ can be estimated as

$$
\bar{\tau}_{i}=\tau_{\mathrm{b}, i}-\tau_{\mathrm{d}, i}+\bar{n}_{i}=\frac{1}{c}\left[\left\|\mathbf{t}_{i}-\mathbf{p}\right\|+\|\tilde{\mathbf{r}}-\mathbf{p}\|-\left\|\mathbf{t}_{i}-\tilde{\mathbf{r}}\right\|\right]+\bar{n}_{i},
$$

where $\bar{n}_{i}$ is the zero-mean Gaussian estimation error due to measurement noise. The Gaussian distribution reasonably models the measurement noise [22] and is commonly in TDOA/TOA measurements (e.g., [9], [10] and [13]). For notational simplicity, we denote $\tau_{i}=c \bar{\tau}_{i}$ and $\tilde{n}_{i}=c \bar{n}_{i}$. In this case, the

\footnotetext{
${ }^{2}$ This is also one of the important features that differentiates the passive radar system from MIMO radar systems where the transmitters and receivers are synchronized [18], [19], i.e., the time of signal transmission is known at the MIMO radar receiver.
} 
ML estimate of the target location is expressed as

$$
\hat{\mathbf{p}}=\arg \min _{\mathbf{p}} \sum_{i=1}^{M}\left[\tilde{\tau}_{i}-\left\|\mathbf{t}_{i}-\mathbf{p}\right\|-\|\tilde{\mathbf{r}}-\mathbf{p}\|\right]^{2},
$$

where

$$
\tilde{\tau}_{i}=\tau_{i}+\left\|\mathbf{t}_{i}-\tilde{\mathbf{r}}\right\|
$$

Note that the knowledge of the true position of the receiver, $\tilde{\mathbf{r}}$, may be inaccurate. Denote the estimated receiver position by $\mathbf{r}$. The relationship between $\tilde{\mathbf{r}}$ and $\mathbf{r}$ is expressed as

$$
\tilde{\mathbf{r}}=\mathbf{r}+\mathbf{e}
$$

where $\mathbf{e}$ is the random estimation error for the receiver position.

\section{Proposed Optimization Approaches}

The unconstrained minimization problem (4) is non-convex. Thus, it is difficult to obtain the global optimum solution with a reasonable complexity. The SDR-based optimization methods applied in $[9,10]$ may not be tight enough in general and, thus, may often fail to provide performance sufficiently close to CRLB. In this section, and motivated by [12], we propose an alternative approach that approximates the ML target localization problem. However, this approximate ML problem is shown to be less sensitive to the SDR and perform better than the methods that solve the exact ML problem. 


\subsection{Optimization without Receiver Position Error}

We first consider the case where the receiver position is exactly known, i.e., $\tilde{\mathbf{r}}=\mathbf{r}_{i}$. Denote $\tau_{i}=\tau_{i}^{\mathrm{o}}+\tilde{n}_{i}$, where $\tau_{i}^{\mathrm{o}}$ is the noise-free observation. Then, from (3), we have

$$
\tau_{i}^{\mathrm{o}}=\left\|\mathbf{t}_{i}-\mathbf{p}\right\|+\|\mathbf{r}-\mathbf{p}\|-\left\|\mathbf{t}_{i}-\mathbf{r}\right\|
$$

or equivalently

$$
\tilde{\tau}_{i}^{\mathrm{o}}-\|\mathbf{r}-\mathbf{p}\|=\left\|\mathbf{t}_{i}-\mathbf{p}\right\|
$$

Squaring both sides of (8) and substituting $\tilde{\tau}_{i}^{o}=\tilde{\tau}_{i}-\tilde{n}_{i}$ into (8), we obtain

$$
\tilde{\tau}_{i}^{2}-2\left(\tilde{\tau}_{i}-q\right) \tilde{n}_{i}+\tilde{n}_{i}^{2}-2 \tilde{\tau}_{i} q+\|\mathbf{r}\|^{2}-\left\|\mathbf{t}_{i}\right\|^{2}-2\left(\mathbf{r}-\mathbf{t}_{i}\right)^{T} \mathbf{p}=0
$$

where $q=\|\mathbf{r}-\mathbf{p}\|$. Neglecting the second-order terms of the noise, $\tilde{n}_{i}^{2}$, and stacking (9) for all $i$, we obtain the following expression

$$
\mathbf{u}-\mathrm{Bz} \approx \mathrm{D} \tilde{\mathbf{n}}
$$

where

$$
\begin{aligned}
& \mathbf{u}=\left[\|\mathbf{r}\|^{2}-\left\|\mathbf{t}_{1}\right\|^{2}+\tilde{\tau}_{1}^{2}, \cdots,\|\mathbf{r}\|^{2}-\left\|\mathbf{t}_{M}\right\|^{2}+\tilde{\tau}_{M}^{2}\right]^{T}, \\
& \mathbf{B}=2\left[\begin{array}{cc}
\left(\mathbf{r}-\mathbf{t}_{1}\right)^{T} & \tilde{\tau}_{1} \\
\left(\mathbf{r}-\mathbf{t}_{2}\right)^{T} & \tilde{\tau}_{2} \\
\vdots & \vdots \\
\left(\mathbf{r}-\mathbf{t}_{M}\right)^{T} & \tilde{\tau}_{M}
\end{array}\right], \\
& \mathbf{D}=2 \operatorname{diag}\left(\tilde{\tau}_{1}-q, \tilde{\tau}_{2}-q, \cdots, \tilde{\tau}_{M}-q\right), \\
& \mathbf{z}=\left[\mathbf{p}^{T}, q\right]^{T}, \\
& \tilde{\mathbf{n}}=\left[\tilde{n}_{1}, \tilde{n}_{2}, \cdots, \tilde{n}_{M}\right]^{T} .
\end{aligned}
$$


By virtue of the underlying NBMF transmission, which exploits non-overlapping spectra, it can be readily assumed that the entries of $\tilde{\mathbf{n}}$ are statistically independent. We further assume that the entries of $\tilde{\mathbf{n}}$ are identically distributed, so that $\tilde{\mathbf{n}}$ is a vector of zero-mean i.i.d. Gaussian random variables with covariance matrix $\sigma_{\mathrm{n}}^{2} \mathbf{I}_{M}$. We maintain that approaches developed in this paper can be easily extended to correlated and non-identically distributed Gaussian noise. From (10), the noise vector can be approximated as

$$
\tilde{\mathbf{n}} \approx \mathrm{D}^{-1}(\mathbf{u}-\mathrm{Bz})
$$

As a result, the ML target localization problem can be approximated as the following minimization problem

$$
\begin{aligned}
& \min _{\mathbf{z}} \frac{1}{\sigma_{\mathrm{n}}^{2}}(\mathbf{u}-\mathbf{B} \mathbf{z})^{T} \mathbf{D}^{-1} \mathbf{D}^{-1}(\mathbf{u}-\mathbf{B} \mathbf{z}) \\
& \text { s.t. } q \triangleq z(n+1)=\|\mathbf{r}-\mathbf{p}\|,
\end{aligned}
$$

where $z(n+1)$ stands for the $(n+1)$ th element of $\mathbf{z}$. After omitting the constant scaling factor $\frac{1}{\sigma_{\mathrm{n}}^{2}}$, (13) can be expressed as

$$
\begin{aligned}
& \min _{\mathbf{z}} \sum_{i=1}^{M} \frac{\left(u_{i}-\mathbf{b}_{i}^{T} \mathbf{z}\right)^{2}}{\left(\tilde{\tau}_{i}-q\right)^{2}} \\
& \text { s.t. } q=\|\mathbf{r}-\mathbf{p}\|,
\end{aligned}
$$

where $u_{i}$ is the $i$ th element of $\mathbf{u}$ and $\mathbf{b}_{i}^{T}$ is the $i$ th row of $\mathbf{B}$. We define $\mathbf{p} \triangleq \tilde{\mathbf{z}}=[z(1), \cdots, z(n)]^{T}, \tilde{\mathbf{b}}_{i}^{T}=\left[b_{i}(1), \cdots, b_{i}(n)\right]$ and $v_{i}=b_{i}(n+1)$, where $b_{i}(k)$ is the $k$ th element of $\mathbf{b}_{i}^{T}$. The objective function (13) can be expressed as

$$
f_{\mathrm{ob}}=\sum_{i=1}^{M}\left[\frac{u_{i}}{\tilde{\tau}_{i}-q}-\frac{\tilde{\mathbf{b}}_{i}^{T} \tilde{\mathbf{z}}}{\tilde{\tau}_{i}-q}-\frac{v_{i} q}{\tilde{\tau}_{i}-q}\right]^{2},
$$


which, after some manipulations, can be expressed as

$$
f_{\mathrm{ob}}=\left[\tilde{\mathbf{z}}^{T}, 1\right] \mathbf{G}(q)\left[\tilde{\mathbf{z}}^{T}, 1\right]^{T}
$$

where the $(n+1) \times(n+1)$ matrix $\mathbf{G}(q)$ is given by

$$
\mathbf{G}(q)=\left[\begin{array}{cc}
\sum_{i=1}^{M} \frac{\tilde{\mathbf{b}}_{i} \tilde{\mathbf{b}}_{i}^{T}}{\left(\tilde{\tau}_{i}-q\right)^{2}} & \sum_{i=1}^{M} \frac{\tilde{\mathbf{b}}_{i}\left(v_{i} q-u_{i}\right)}{\left(\tilde{\tau}_{i}-q\right)^{2}} \\
\sum_{i=1}^{M} \frac{\tilde{\mathbf{b}}_{i}^{T}\left(v_{i} q-u_{i}\right)}{\left(\tilde{\tau}_{i}-q\right)^{2}} & \sum_{i=1}^{M} \frac{\left(v_{i} q-u_{i}\right)^{2}}{\left(\tilde{\tau}_{i}-q\right)^{2}}
\end{array}\right] .
$$

Thus, the minimization problem (14) is given by

$$
\begin{aligned}
& \min _{\tilde{\mathbf{z}}, q} \operatorname{tr}\left\{\left[\begin{array}{c}
\tilde{\mathbf{z}} \\
1
\end{array}\right]\left[\tilde{\mathbf{z}}^{T}, 1\right] \mathbf{G}(q)\right\} \\
& \text { s.t. } q^{2}=\|\mathbf{r}-\mathbf{p}\|^{2} \longleftrightarrow q^{2}=\operatorname{tr}\left\{\left[\begin{array}{l}
\tilde{\mathbf{z}} \\
1
\end{array}\right]\left[\tilde{\mathbf{z}}^{T}, 1\right]\left[\begin{array}{cc}
\mathbf{I}_{n} & -\mathbf{r} \\
-\mathbf{r}^{T} & \mathbf{r}^{T} \mathbf{r}
\end{array}\right]\right\},
\end{aligned}
$$

which is clearly a non-convex optimization problem. We define $\tilde{\mathbf{Z}}=\tilde{\mathbf{z}} \tilde{\mathbf{Z}}^{T}$, which is relaxed as $\tilde{\mathbf{Z}} \succeq \tilde{\mathbf{z}} \tilde{\mathbf{Z}}^{T}$, i.e., $\tilde{\mathbf{Z}}-\tilde{\mathbf{z}} \tilde{\mathbf{z}}^{T}$ is positive semi-definite. This yields the following optimization problem

$$
\begin{aligned}
& \min _{\tilde{\mathbf{z}}, \tilde{\mathbf{Z}}, q} \operatorname{tr}\left\{\left[\begin{array}{cc}
\tilde{\mathbf{Z}} & \tilde{\mathbf{Z}} \\
\tilde{\mathbf{z}}^{T} & 1
\end{array}\right] \mathbf{G}(q)\right\} \\
& \text { s.t. } q^{2}=\operatorname{tr}\left\{\left[\begin{array}{cc}
\tilde{\mathbf{Z}} & \tilde{\mathbf{z}} \\
\tilde{\mathbf{z}}^{T} & 1
\end{array}\right]\left[\begin{array}{cc}
\mathbf{I}_{n} & -\mathbf{r} \\
-\mathbf{r}^{T} & \mathbf{r}^{T} \mathbf{r}
\end{array}\right]\right\}, \\
& {\left[\begin{array}{cc}
1 & \tilde{\mathbf{z}}^{T} \\
\tilde{\mathbf{z}} & \tilde{\mathbf{Z}}
\end{array}\right] \succeq 0 .}
\end{aligned}
$$

For a given $q$, (19) is a convex optimization problem w.r.t. $\tilde{\mathbf{z}}$ and $\tilde{\mathbf{Z}}$. However, the joint optimization problem (w.r.t. $q, \tilde{\mathbf{z}}$ and $\tilde{\mathbf{Z}}$ ) is not convex. On the 
other hand, since $q$ is a scalar (also positive), it is still easy to find the global optimum solution of (19) by using an exhaustive search over $q$, where (19) is solved over a fine grid of $q$ and $\tilde{\mathbf{z}}$ is selected for which the objective function is minimum. To reduce the complexity of solving the optimum value of $q$, we propose to employ a line search (e.g., bisection search) over $q$ for solving the optimization problem (19). Since it is very difficult to analytically prove that the bisection search guarantees the global minimum for (19), we pursue a numerical approach. Let $\left[\tilde{\mathbf{z}}_{j}^{*}, \tilde{\mathbf{Z}}_{j}^{*}\right]$ be an optimal solution of (19) for a given $q=q_{j}$, where $j=\{1, \cdots, J\}$. Let $\tilde{f}_{\mathrm{ob}}(q)=\left\{f_{\mathrm{ob}}\left(\tilde{\mathbf{z}}_{j}^{*}, \tilde{\mathbf{Z}}_{j}^{*}, q_{j}\right)\right\}_{j=1}^{J}$ be the resulting function w.r.t. $\left\{q_{j}\right\}$. In the considered simulation scenario (see Fig. 2 of Section 5) with perfectly known receiver position and an arbitrary noise realization, it can be observed from Fig. 1 that $\tilde{f}_{\mathrm{ob}}(q)$ is an unimodal function. This suggests that the bisection approach accurately finds the global minimum in (19).

Assume that the optimum $q$ lies in the interval $\left[q_{1}, q_{\mathrm{u}}\right]$. It is obvious in the underlying problem that $q_{1}=0$. The algorithm (Algorithm 1) for solving (19) is then provided below.

1) Initialize $q_{\mathrm{l}}, q_{\mathrm{u}}$ and set $\epsilon>0$.

2) Solve (19) with $q=q_{\mathrm{l}}$ and $q=q_{\mathrm{u}}$.

3) If $f_{\mathrm{ob}}\left(q_{\mathrm{l}}\right)<f_{\mathrm{ob}}\left(q_{\mathrm{u}}\right)$, set $q_{\mathrm{u}}=\frac{q_{\mathrm{l}}+q_{\mathrm{u}}}{2}$; otherwise set $q_{\mathrm{l}}=\frac{q_{\mathrm{l}}+q_{\mathrm{u}}}{2}$.

4) Go to step 2 until $\left|q_{\mathrm{u}}-q_{1}\right| \leq \epsilon$.

Remark 1: Let $\left[\tilde{\mathbf{z}}^{*}, \tilde{\mathbf{Z}}^{*}, q^{*}\right]$ be an optimal solution of the problem (19). 
Notice that the number of equality constraints is $L=1$. According to the Shapiro-Barvinok-Pataki (SBP) result [23], there exists an optimal solution $\tilde{\mathbf{Z}}^{*} \operatorname{such}$ that $\operatorname{rank}\left(\tilde{\mathbf{Z}}^{*}\right)\left(\operatorname{rank}\left(\tilde{\mathbf{Z}}^{*}\right)+1\right) \leq 2 L$. Since $L=1$ in (19) and $\operatorname{rank}\left(\tilde{\mathbf{Z}}^{*}\right) \neq 0$, we find that $\tilde{\mathbf{Z}}^{*}$ is rank-one.

The CRLB for the case without receiver position error is given by (see Appendix 7.1)

$$
\mathrm{CRB}=\operatorname{tr}\left(\mathbf{F}^{-1}\right)
$$

where

$$
\begin{gathered}
\mathbf{F}=\frac{1}{\sigma_{\mathrm{n}}^{2}} \frac{\partial \tilde{\mathbf{s}}^{T}}{\partial \mathbf{p}} \mathbf{E}^{T} \mathbf{E} \frac{\partial \tilde{\mathbf{s}}}{\partial \mathbf{p}^{T}}, \frac{\partial \tilde{\mathbf{s}}^{T}}{\partial \mathbf{p}}=\left[\frac{\mathbf{r}-\mathbf{p}}{\|\mathbf{r}-\mathbf{p}\|}, \frac{\mathbf{t}_{1}-\mathbf{p}}{\left\|\mathbf{t}_{1}-\mathbf{p}\right\|}, \cdots, \frac{\mathbf{t}_{M}-\mathbf{p}}{\left\|\mathbf{t}_{M}-\mathbf{p}\right\|}\right],(21) \\
\tilde{\mathbf{s}}=\left[q=\|\mathbf{r}-\mathbf{p}\|, s_{1}=\left\|\mathbf{t}_{1}-\mathbf{p}\right\|, \cdots, s_{M}=\left\|\mathbf{t}_{M}-\mathbf{p}\right\|\right]^{T}, \mathbf{E}=\left[\mathbf{1}_{M}, \mathbf{I}_{M}\right] .
\end{gathered}
$$

\subsection{Optimization with Receiver Position Error}

In the presence of random position error (i.e., $\tilde{\mathbf{r}}=\mathbf{r}+\mathbf{e}$ ), we can express the measured range difference for the signal of $i$ th illuminator as

$$
\tau_{i}^{\mathrm{r}}=\left\|\mathbf{t}_{i}-\mathbf{p}\right\|+\|(\mathbf{r}+\mathbf{e})-\mathbf{p}\|-\left\|(\mathbf{r}+\mathbf{e})-\mathbf{t}_{i}\right\|+\tilde{n}_{i} .
$$

We consider that $\mathbf{e}$ is small when compared to $(\mathbf{r}-\mathbf{p})$ and $\left(\mathbf{r}-\mathbf{t}_{i}\right), \forall i$, i.e., $\|\mathbf{e}\| \ll\|\mathbf{r}-\mathbf{p}\|,\left\|\mathbf{r}-\mathbf{t}_{i}\right\|, \forall i$. Using Taylor's series expansion, we get

$$
\begin{aligned}
& \|(\mathbf{r}+\mathbf{e})-\mathbf{p}\|=\|\mathbf{r}-\mathbf{p}\|+\mathbf{e}^{T} \frac{\mathbf{r}-\mathbf{p}}{\|\mathbf{r}-\mathbf{p}\|}+\mathcal{O}(\|\mathbf{e}\|) \\
& \left\|(\mathbf{r}+\mathbf{e})-\mathbf{t}_{i}\right\|=\left\|\mathbf{r}-\mathbf{t}_{i}\right\|+\mathbf{e}^{T} \frac{\mathbf{r}-\mathbf{t}_{i}}{\left\|\mathbf{r}-\mathbf{t}_{i}\right\|}+\mathcal{O}(\|\mathbf{e}\|),
\end{aligned}
$$

where $\mathcal{O}(\|\mathbf{e}\|)$ stands for higher-order terms of $\|\mathbf{e}\|$. Substituting (23) into (22), we obtain

$$
\tau_{i}^{\mathrm{r}} \approx\left\|\mathbf{t}_{i}-\mathbf{p}\right\|+\|\mathbf{r}-\mathbf{p}\|-\left\|\mathbf{r}-\mathbf{t}_{i}\right\|+\mathbf{e}^{T}\left[\frac{\mathbf{r}-\mathbf{p}}{\|\mathbf{r}-\mathbf{p}\|}-\frac{\mathbf{r}-\mathbf{t}_{i}}{\left\|\mathbf{r}-\mathbf{t}_{i}\right\|}\right]+\tilde{n}_{i} .
$$


Define $\tilde{\tau}_{i}^{\mathrm{r}}=\tau_{i}^{\mathrm{r}}+\left\|\mathbf{r}-\mathbf{t}_{i}\right\|, \mathbf{w}=\frac{\mathbf{r}-\mathbf{p}}{\|\mathbf{r}-\mathbf{p}\|}, \mathbf{w}_{i}=\frac{\mathbf{r}-\mathbf{t}_{i}}{\left\|\mathbf{r}-\mathbf{t}_{i}\right\|}$ and $\tilde{\mathbf{w}}_{i}=\mathbf{w}-\mathbf{w}_{i}$. Then, (24) can be expressed as

$$
\left(\tilde{\tau}_{i}^{\mathrm{r}}-\tilde{n}_{i}\right)-\|\mathbf{r}-\mathbf{p}\|-\mathbf{e}^{T} \tilde{\mathbf{w}}_{i} \approx\left\|\mathbf{t}_{i}-\mathbf{p}\right\|
$$

Squaring both sides of (25) and after some derivations, we get

$$
\begin{aligned}
& \left(\tilde{\tau}_{i}^{\mathrm{r}}\right)^{2}+\|\mathbf{r}\|^{2}-\left\|\mathbf{t}_{i}\right\|^{2}-2\left(\mathbf{r}_{i}-\mathbf{t}\right)^{T} \mathbf{p}-2 \tilde{\tau}_{i}^{\mathrm{r}} q+\tilde{n}_{i}^{2}+2 \tilde{n}_{i} \mathbf{e}^{T} \tilde{\mathbf{w}}_{i} \\
& +\mathbf{e}^{T} \tilde{\mathbf{w}}_{i} \tilde{\mathbf{w}}_{i} \mathbf{e} \approx 2 \tilde{n}_{i}\left(\tilde{\tau}_{i}^{\mathrm{r}}-q\right)+2\left(\tilde{\tau}_{i}^{\mathrm{r}}-q\right) \mathbf{e}^{T} \tilde{\mathbf{w}}_{i} .
\end{aligned}
$$

Neglecting the second order terms of noise and the cross-order term between noise and position error, for $i=1, \cdots, M,(26)$ can be expressed in the vector form as

$$
\mathbf{u}-\mathrm{Bz} \approx \mathrm{D} \tilde{\mathbf{n}}+\mathrm{Ce}
$$

where

$$
\mathbf{C}=2\left[\begin{array}{c}
\left(\tilde{\tau}_{1}^{\mathrm{r}}-q\right) \tilde{\mathbf{w}}_{1}^{T} \\
\left(\tilde{\tau}_{2}^{\mathrm{r}}-q\right) \tilde{\mathbf{w}}_{2}^{T} \\
\vdots \\
\left(\tilde{\tau}_{M}^{\mathrm{r}}-q\right) \tilde{\mathbf{w}}_{M}^{T}
\end{array}\right]
$$

Notice that (27) can be expressed as

$$
\mathrm{D}^{-1}(\mathbf{u}-\mathbf{B z}) \approx \tilde{\mathbf{n}}+\mathbf{D}^{-1} \mathbf{C e}
$$

Assume that the position error e is Gaussian distributed with zero-mean and covariance matrix $\mathbf{Q}$. Since e and $\tilde{\mathbf{n}}$ are independent, we obtain

$$
\mathrm{E}\left\{\left(\tilde{\mathbf{n}}+\mathbf{D}^{-1} \mathbf{C e}\right)\left(\tilde{\mathbf{n}}+\mathbf{D}^{-1} \mathbf{C e}\right)^{T}\right\}=\sigma_{\mathrm{n}}^{2} \mathbf{I}_{M}+\mathbf{D}^{-1} \mathbf{C Q C}^{T}\left(\mathbf{D}^{-1}\right)^{T}
$$


Particularly, under the assumption $\mathbf{Q}=\sigma_{\mathrm{e}}^{2} \mathbf{I}_{n}$, where $\sigma_{\mathrm{e}}^{2}$ is the variance of the elements of $\mathbf{e}$, the ML localization problem can be approximately expressed as

$$
\begin{aligned}
& \min _{\mathbf{z}}\left\{(\mathbf{u}-\mathbf{B} \mathbf{z})^{T} \mathbf{D}^{-1}\left(\sigma_{\mathrm{n}}^{2} \mathbf{I}_{M}+\sigma_{\mathrm{e}}^{2} \mathbf{D}^{-1} \mathbf{C C}^{T} \mathbf{D}^{-1}\right)^{-1} \mathbf{D}^{-1}(\mathbf{u}-\mathbf{B z})\right\} \\
& \text { s.t. } q \triangleq z(n+1)=\|\mathbf{r}-\mathbf{p}\| .
\end{aligned}
$$

The objective function of the optimization problem (31) can be simplified to

$$
f_{\mathrm{rob}}=\frac{1}{4}(\mathbf{u}-\mathbf{B} \mathbf{z})^{T}\left(\sigma_{\mathrm{n}}^{2} \mathbf{D} \mathbf{D}^{T}+\sigma_{\mathrm{e}}^{2} \mathbf{C C}^{T}\right)^{-1}(\mathbf{u}-\mathbf{B} \mathbf{z})
$$

where

$$
\mathbf{D D}^{T}=\operatorname{diag}\left(\left(\tilde{\tau}_{1}^{\mathrm{r}}-q\right)^{2},\left(\tilde{\tau}_{2}^{\mathrm{r}}-q\right)^{2}, \cdots,\left(\tilde{\tau}_{M}^{\mathrm{r}}-q\right)^{2}\right)
$$

and

$$
\mathbf{C C}^{T}=\left[\begin{array}{cccc}
\left(\tilde{\tau}_{1}^{\mathrm{r}}-q\right)^{2} \tilde{\mathbf{w}}_{1}^{T} \tilde{\mathbf{w}}_{1} & \left(\tilde{\tau}_{1}^{\mathrm{r}}-q\right)\left(\tilde{\tau}_{2}^{\mathrm{r}}-q\right) \tilde{\mathbf{w}}_{1}^{T} \tilde{\mathbf{w}}_{2} & \cdots & \left(\tilde{\tau}_{1}^{\mathrm{r}}-q\right)\left(\tilde{\tau}_{M}^{\mathrm{r}}-q\right) \tilde{\mathbf{w}}_{1}^{T} \tilde{\mathbf{w}}_{M} \\
\left(\tilde{\tau}_{2}^{\mathrm{r}}-q\right)\left(\tilde{\tau}_{1}^{\mathrm{r}}-q\right) \tilde{\mathbf{w}}_{2}^{T} \tilde{\mathbf{w}}_{1} & \left(\tilde{\tau}_{2}^{\mathrm{r}}-q\right)^{2} \tilde{\mathbf{w}}_{2}^{T} \tilde{\mathbf{w}}_{2} & \cdots & \left(\tilde{\tau}_{2}^{\mathrm{r}}-q\right)\left(\tilde{\tau}_{M}^{\mathrm{r}}-q\right) \tilde{\mathbf{w}}_{2}^{T} \tilde{\mathbf{w}}_{M} \\
\vdots & \vdots & \vdots & \vdots \\
\left(\tilde{\tau}_{M}^{\mathrm{r}}-q\right)\left(\tilde{\tau}_{1}^{\mathrm{r}}-q\right) \tilde{\mathbf{w}}_{M}^{T} \tilde{\mathbf{w}}_{1} & \left(\tilde{\tau}_{M}^{\mathrm{r}}-q\right)\left(\tilde{\tau}_{2}^{\mathrm{r}}-q\right) \tilde{\mathbf{w}}_{M}^{T} \tilde{\mathbf{w}}_{2} & \cdots & \left(\tilde{\tau}_{M}^{\mathrm{r}}-q\right)^{2} \tilde{\mathbf{w}}_{M}^{T} \tilde{\mathbf{w}}_{M}
\end{array}\right] .
$$

Since $\mathbf{w}^{T} \mathbf{w}=1$ and $\mathbf{w}_{i}^{T} \mathbf{w}_{i}=1, \forall i$, we have

$$
\begin{aligned}
\tilde{\mathbf{w}}_{i}^{T} \tilde{\mathbf{w}}_{i} & =2\left[1-\frac{(\mathbf{r}-\mathbf{p})^{T}}{q} \mathbf{w}_{i}\right], \forall i \\
\tilde{\mathbf{w}}_{i}^{T} \tilde{\mathbf{w}}_{k} & =1-\frac{(\mathbf{r}-\mathbf{p})^{T}}{q}\left(\mathbf{w}_{i}+\mathbf{w}_{k}\right)+\mathbf{w}_{i}^{T} \mathbf{w}_{k}, \forall i, k, i \neq k .
\end{aligned}
$$

Introducing an auxiliary variable $\gamma>0$ and defining

$$
\tilde{\mathbf{B}} \triangleq \mathbf{B}_{[1: M, 1: n]}, \quad \tilde{\mathbf{b}} \triangleq \mathbf{B}_{[1: M, n+1]}, \mathbf{p} \triangleq \tilde{\mathbf{z}}
$$


(31) can be expressed as

$$
\begin{aligned}
& \min _{\{\tilde{\mathbf{z}}, q, \gamma\}} \gamma \\
& \text { s.t. } \gamma \geq(\mathbf{u}-\tilde{\mathbf{B}} \tilde{\mathbf{z}}-\tilde{\mathbf{b}} q)^{T}\left(\sigma_{\mathrm{n}}^{2} \mathbf{D} \mathbf{D}^{T}+\sigma_{\mathrm{e}}^{2} \mathbf{C} \mathbf{C}^{T}\right)^{-1}(\mathbf{u}-\tilde{\mathbf{B}} \tilde{\mathbf{z}}-\tilde{\mathbf{b}} q), \\
& \qquad q^{2}=\mathbf{r}^{T} \mathbf{r}-2 \mathbf{r}^{T} \tilde{\mathbf{z}}+\tilde{\mathbf{z}}^{T} \tilde{\mathbf{z}}
\end{aligned}
$$

Using the Schur-complement theorem [24], the inequality constraint of (37) can be expressed as

$$
\left[\begin{array}{cc}
\sigma_{\mathrm{n}}^{2} \mathbf{D D}^{T}+\sigma_{\mathrm{e}}^{2} \mathbf{C} \mathbf{C}^{T} & (\mathbf{u}-\tilde{\mathbf{B}} \tilde{\mathbf{z}}-\tilde{\mathbf{b}} q) \\
(\mathbf{u}-\tilde{\mathbf{B}} \tilde{\mathbf{z}}-\tilde{\mathbf{b}} q)^{T} & \gamma
\end{array}\right] \succeq 0
$$

Defining $\bar{z}=\tilde{\mathbf{z}}^{T} \tilde{\mathbf{z}}$ and using the relaxation $\bar{z} \geq \tilde{\mathbf{z}}^{T} \tilde{\mathbf{z}}$, the optimization problem (37) can be expressed as

$$
\begin{aligned}
& \min _{\{\tilde{\mathbf{z}}, q, \gamma, \bar{z}\}} \gamma \\
& \text { s.t. }\left[\begin{array}{rc}
\sigma_{\mathrm{n}}^{2} \mathbf{D} \mathbf{D}^{T}+\sigma_{\mathrm{e}}^{2} \mathbf{C C}^{T} & (\mathbf{u}-\tilde{\mathbf{B}} \tilde{\mathbf{z}}-\tilde{\mathbf{b}} q) \\
(\mathbf{u}-\tilde{\mathbf{B}} \tilde{\mathbf{z}}-\tilde{\mathbf{b}} q)^{T} & \gamma
\end{array}\right] \succeq 0 \\
& q^{2}=\mathbf{r}^{T} \mathbf{r}-2 \mathbf{r}^{T} \tilde{\mathbf{z}}+\bar{z}, \\
& {\left[\begin{array}{cc}
\mathbf{I}_{n} & \tilde{\mathbf{z}} \\
\tilde{\mathbf{z}}^{T} & \bar{z}
\end{array}\right] \succeq 0 .}
\end{aligned}
$$

For a given $q$, the optimization problem (39) is convex. The joint optimization is then solved in conjunction with the bisection search over $q$. The algorithm (Algorithm 1), as shown for the case without position errors, can then be applied to solve (39).

When the assumed receiver position is subject to errors, the CRLB can 
be expressed as (see Appendix 7.2)

$$
\widetilde{C R B}=\operatorname{tr}\left(\left(\mathbf{F}_{\mathbf{p}}-\mathbf{F}_{\mathbf{p} \tilde{\mathbf{r}}} \mathbf{F}_{\tilde{\mathbf{r}}}^{-1} \mathbf{F}_{\mathbf{p} \tilde{\mathbf{r}}}^{T}\right)^{-1}\right)
$$

where $\mathbf{F}_{\mathbf{p}}=\mathbf{F}$,

$$
\begin{aligned}
\mathbf{F}_{\mathbf{p} \tilde{\mathbf{r}}} & =\frac{1}{\sigma_{\mathrm{n}}^{2}} \frac{\partial \tilde{\mathbf{s}}^{T}}{\partial \mathbf{p}} \mathbf{E}^{T} \mathbf{E} \frac{\partial \tilde{\mathbf{s}}}{\partial \tilde{\mathbf{r}}^{T}}, \\
\mathbf{F}_{\tilde{\mathbf{r}}} & =\frac{1}{\sigma_{\mathrm{n}}^{2}} \frac{\partial \tilde{\mathbf{s}}^{T}}{\partial \tilde{\mathbf{r}}} \mathbf{E}^{T} \mathbf{E} \frac{\partial \tilde{\mathbf{s}}}{\partial \tilde{\mathbf{r}}^{T}}, \\
\frac{\partial \tilde{\mathbf{s}}^{T}}{\partial \tilde{\mathbf{r}}} & =\left[\frac{\tilde{\mathbf{r}}-\mathbf{p}}{\|\tilde{\mathbf{r}}-\mathbf{p}\|}, \mathbf{0}_{1 \times n}, \cdots, \mathbf{0}_{1 \times n}\right] .
\end{aligned}
$$

\section{Complexity Analysis}

We present the computational complexity of the proposed optimization algorithms using the approach in [25]. For a given $q$, the number of iterations required for solving $(19)$ is upper bounded by $\tilde{\mathcal{O}}\left((n+1)^{\frac{1}{2}}\right)$, whereas the work load per iteration is upper bounded by $\tilde{\mathcal{O}}\left(\left(n^{2}+n\right)^{2}(n+1)^{2}\right)$. The bisection search w.r.t. $q$ requires $\bar{L}=\log _{2}\left(\frac{q_{\mathrm{u}}-q_{1}}{\epsilon}\right)$ iterations. This means that the overall complexity for the case without position errors is approximately $\tilde{\mathcal{O}}\left(n^{6.5} \bar{L}\right)$. It is interesting to note that the complexity of Algorithm 1 does not depend on $M$. In a similar manner, for a given $q$, we can show that the complexity of (39) in terms of number of iterations is $\tilde{\mathcal{O}}\left((n+M+2)^{\frac{1}{2}}\right)$, whereas the complexity per iteration is $\tilde{\mathcal{O}}\left((n+2)^{2}\left((n+1)^{2}+(M+1)^{2}\right)\right)$. This means that the total complexity of (39) is approximately $\tilde{\mathcal{O}}\left((n+M)^{0.5} n^{2}\left(n^{2}+M^{2}\right)\right)$. For the case without position errors, the SDP approach (before local optimization) of [9] requires $\tilde{\mathcal{O}}\left((n+M+3)^{\frac{1}{2}}\right)$ iterations, where the complexity per-iteration is given by $\tilde{\mathcal{O}}\left(\left((M+1)^{2}+M+n+2\right)^{2}\left((M+2)^{2}+(n+1)^{2}\right)\right)$. 
For a gradient-based local optimization, such as steepest-descent method, $\tilde{\mathcal{O}}\left(\epsilon^{-2}\right)$ iterations are required to keep the norm of the gradient below $\epsilon[26]$.

Thus, the total complexity is given by $\tilde{\mathcal{O}}\left((n+M)^{0.5}\left(M^{2}+M+n\right)^{2}\left(M^{2}+n^{2}\right)\right)$ plus $\tilde{\mathcal{O}}\left(\epsilon^{-2}\right)$. On the other hand, for the case with position errors, the SDP approach (before local optimization) in [9] requires $\tilde{\mathcal{O}}\left((n+1+2(M+2))^{\frac{1}{2}}\right)$ iterations. The corresponding computational complexity of each iteration is given by $\tilde{\mathcal{O}}\left(\left((M+1)^{2}+M+n+4\right)^{2}\left(2(M+2)^{2}+(n+1)^{2}\right)\right)$, which results in a total complexity of $\tilde{\mathcal{O}}\left((n+2 M)^{0.5}\left(M^{2}+M+n\right)^{2}\left(2 M^{2}+n^{2}\right)\right)+\tilde{\mathcal{O}}\left(\epsilon^{-2}\right)$. Note that in practice $n<<M$. For this case, the total complexity between the proposed and SDP methods is compared in Table 1.

It can be observed from this table that, as long as $\bar{L} \leq\left(\frac{M}{n}\right)^{6.5}$ for the case without errors and $\bar{L} \leq\left(\frac{M^{2}}{n}\right)^{2}$ for the case with errors, the complexity of the proposed method does not exceed that of the SDP.

\section{Numerical results}

Computer simulations are conducted to demonstrate the effectiveness of the proposed method. The stationary target and receiver are located at positions $\mathbf{p}=[600,550]^{T}$ meters and $\mathbf{r}=[-200,10]$ meters, respectively. The illuminators are located at the following positions

$$
\left[\begin{array}{ccccccc}
800 & 900 & 1000 & 1100 & 1100 & 1100 & 1100 \\
200 & 200 & 200 & 200 & 300 & 400 & 500
\end{array}\right] \text { meters. }
$$

A two-dimensional coordinate system for the locations of the target, illuminators and receiver is shown in Fig. 2. 
The CVX toolbox [27] is used to solve the convex optimization problems (19) and (39). These optimization problems are solved within a framework of bisection algorithm outlined in Algorithm 1. We run Algorithm 1 and corresponding algorithm for (39) by taking $q_{\mathrm{l}}=2 \mathrm{~m}, q_{\mathrm{u}}=1000 \mathrm{~m}$ and $\epsilon=1$ m. Note that smaller values of $\epsilon$ can be taken for improving convergence accuracy, whereas larger values of $q_{u}$ can be taken if we do not have even a coarse knowledge of the target-receiver range. Both settings in general result in higher computational complexity, since the bisection search requires more iterations. We compare the proposed method with both the SDP method that employs local optimization [9] and the LLS method. Notice that the SDP method [9] without local optimization gives very poor results in our simulation scenarios, and thus, only the results after local optimization are shown. As suggested in [9], the penalty parameter for this method is varied between $10^{-3}$ and $10^{-7}$. The solution of the LLS method at a given estimate $\mathbf{p}_{0}$ is given by

$$
\mathbf{p}=\left(\mathbf{A}^{T} \mathbf{A}\right)^{-1} \mathbf{A}^{T}\left(\boldsymbol{\tau}^{\mathrm{mo}}-\mathbf{h}\right),
$$


where

$$
\begin{gathered}
\mathbf{A}=\left[\begin{array}{c}
\mathbf{a}_{1}^{T} \triangleq \frac{\left(\mathbf{p}_{0}-\mathbf{t}_{1}\right)^{T}}{\left\|\mathbf{p}_{0}-\mathbf{t}_{1}\right\|}+\frac{\left(\mathbf{p}_{0}-\tilde{\mathbf{r}}\right)^{T}}{\left\|\mathbf{p}_{0}-\tilde{\mathbf{r}}\right\|} \\
\vdots \\
\mathbf{a}_{M}^{T} \triangleq \frac{\left(\mathbf{p}_{0}-\mathbf{t}_{M}\right)^{T}}{\left\|\mathbf{p}_{0}-\mathbf{t}_{M}\right\|}+\frac{\left(\mathbf{p}_{0}-\tilde{\mathbf{r}}\right)^{T}}{\left\|\mathbf{p}_{0}-\tilde{\mathbf{r}}\right\|}
\end{array}\right] \\
\mathbf{h}=\left[\begin{array}{c}
\left\|\mathbf{p}_{0}-\mathbf{t}_{1}\right\|+\left\|\mathbf{p}_{0}-\tilde{\mathbf{r}}\right\|-\left\|\mathbf{t}_{1}-\tilde{\mathbf{r}}\right\|+\mathbf{p}_{0}^{T} \mathbf{a}_{1} \\
\vdots \\
\left\|\mathbf{p}_{0}-\mathbf{t}_{M}\right\|+\left\|\mathbf{p}_{0}-\tilde{\mathbf{r}}\right\|-\left\|\mathbf{t}_{M}-\tilde{\mathbf{r}}\right\|+\mathbf{p}_{0}^{T} \mathbf{a}_{M}
\end{array}\right], \\
\boldsymbol{\tau}^{\mathrm{mo}}=\left[\tau_{1}, \cdots, \tau_{M}\right]^{T} \text { or }\left[\tau_{1}^{\mathrm{r}}, \cdots, \tau_{M}^{\mathrm{r}}\right]^{T} .
\end{gathered}
$$

For the LLS method, we take an initial estimate as $\mathbf{p}_{0}=[10,10]^{T} \mathrm{~m}^{3}$, determine $\mathbf{p}$ using (42), and update $\mathbf{p}_{0}$ iteratively until $\left\|\mathbf{p}-\mathbf{p}_{0}\right\| \leq 1 \mathrm{~m}$. We first consider the case in which the observed data set is contaminated by measurement noise, whereas the receiver position is perfectly known. The measurement noise follows a zero-mean Gaussian distribution with a variance of $\sigma_{\mathrm{n}}^{2}$. All results are obtained from $N=200$ independent realizations of noise and position error.

Fig. 3 shows the root mean-square error (RMSE) of the estimated target position as a function of $\sigma_{\mathrm{n}}$. The RMSE is defined as

$$
\operatorname{RMSE}=\sqrt{\frac{1}{N} \sum_{n=1}^{N}\left\|\hat{\mathbf{p}}^{(n)}-\mathbf{p}\right\|^{2}},
$$

\footnotetext{
${ }^{3}$ We also tested the LLS method with other values of $\mathbf{p}_{0}$ but found that the performance does not improve w.r.t. other methods, especially at higher values of noise. As a result, for the sake of conciseness, we show the LLS performance using only the abovementioned value of $\mathbf{p}_{0}$.
} 
where $\hat{\mathbf{p}}^{(n)}$ is the estimated target position for the $n$th simulation and $\mathbf{p}$ is the actual target position. The performance of the proposed method is compared with the SDP method that refines its solution with local optimization. The RMSE performance of the LLS method is also shown in Fig. 3. It is observed from this figure that the proposed method outperforms both SDP and LLS methods and provides performance very close to the CRLB. For $\sigma_{\mathrm{n}} \geq 38 \mathrm{~m}$, the performance of the LLS approach deviates much faster from CRLB than the other two methods.

In Fig. 4, the performance of the proposed robust method is depicted for the case where the receiver position is subject to a position error, the elements of which are independent and uniformly distributed between $-d \mathrm{~m}$ and $+d \mathrm{~m}$. As in the case without position error, RMSE of the LLS method starts to rise rapidly for $\sigma_{\mathrm{n}} \geq 38 \mathrm{~m}$, whereas that of the proposed method and SDP (with local optimization) remain stable. It can be observed from Fig. 4 that the proposed method provides better RMSE performance than the SDP method and overall performance very close to the CRLB. It is important to emphasize that the results for SDP method without local optimization are not shown since such approach fails to produce reasonable results for both the cases with and without the receiver position error.

\section{Conclusions}

We considered the problem of localizing a target using time-of-arrival information measured at a receiver from the signals of multiple illuminators in a multistatic passive radar system. The localization problems are formulated 
using an approximate maximum likelihood estimate of the target location. The resulting non-convex problems are reformulated as convex problems using the semi-definite relaxation approach and solved in a framework of bisection algorithm. The optimization problems are examined for the cases when only measurement errors are present and when both the measurement and receiver position errors are present. Simulation results verify the superior performance of the proposed method over the semidefinite programming method based on exact maximum likelihood estimate of the target location.

\section{Appendix}

\subsection{Derivation of $C R L B$ when receiver position is perfectly known}

The log-likelihood function can be expressed as

$$
\begin{aligned}
L(\mathbf{p}) & =-\frac{1}{2 \sigma_{\mathrm{n}}^{2}} \sum_{i=1}^{M}\left(\tilde{\tau}_{i}-\left(q+s_{i}\right)\right)^{2}, s_{i} \triangleq\left\|\mathbf{t}_{i}-\mathbf{p}\right\|, \forall i, \\
& =-\frac{1}{2 \sigma_{\mathrm{n}}^{2}}\left\{\|\tilde{\boldsymbol{\tau}}\|^{2}-2 \overline{\boldsymbol{\tau}}^{T} \tilde{\mathbf{s}}+\tilde{\mathbf{s}}^{T} \mathbf{E}^{T} \mathbf{E} \tilde{\mathbf{s}}\right\},
\end{aligned}
$$

where

$$
\begin{aligned}
& \tilde{\boldsymbol{\tau}}=\left[\tilde{\tau}_{1}, \cdots, \tilde{\tau}_{M}\right]^{T}, \overline{\boldsymbol{\tau}}^{T}=\left[\sum_{i} \tilde{\tau}_{i}, \tilde{\boldsymbol{\tau}}^{T}\right], \\
& \tilde{\mathbf{s}}=\left[q, s_{1}, \cdots, s_{M}\right]^{T}, \mathbf{E}=\left[\mathbf{1}_{M}, \mathbf{I}_{M}\right] .
\end{aligned}
$$

The Fisher information matrix is given by

$$
\mathbf{F}=-\left[\begin{array}{cccc}
\mathrm{E}\left\{\frac{\partial^{2} L(\mathbf{p})}{\partial p_{1}^{2}}\right\} & \mathrm{E}\left\{\frac{\partial^{2} L(\mathbf{p})}{\partial p_{1} \partial p_{2}}\right\} & \cdots & \mathrm{E}\left\{\frac{\partial^{2} L(\mathbf{p})}{\partial p_{1} \partial p_{n}}\right\} \\
\mathrm{E}\left\{\frac{\partial^{2} L(\mathbf{p})}{\partial p_{2} \partial p_{1}}\right\} & \mathrm{E}\left\{\frac{\partial^{2} L(\mathbf{p})}{\partial p_{2}^{2}}\right\} & \cdots & \mathrm{E}\left\{\frac{\partial^{2} L(\mathbf{p})}{\partial p_{2} \partial p_{n}}\right\} \\
\vdots & \vdots & \vdots & \vdots \\
\mathrm{E}\left\{\frac{\partial^{2} L(\mathbf{p})}{\partial p_{n} \partial p_{1}}\right\} & \mathrm{E}\left\{\frac{\partial^{2} L(\mathbf{p})}{\partial p_{n} \partial p_{2}}\right\} & \cdots & \mathrm{E}\left\{\frac{\partial^{2} L(\mathbf{p})}{\partial p_{n}^{2}}\right\}
\end{array}\right],
$$


where $p_{l}$ denotes the $l$ th element of $\mathbf{p}$. Since

$$
\frac{\partial L(\mathbf{p})}{\partial p_{l}}=-\frac{1}{\sigma_{\mathrm{n}}^{2}}\left\{-\overline{\boldsymbol{\tau}}^{T} \frac{\partial \tilde{\mathbf{s}}}{\partial p_{l}}+\tilde{\mathbf{s}}^{T} \mathbf{E}^{T} \mathbf{E} \frac{\partial \tilde{\mathbf{s}}}{\partial p_{l}}\right\},
$$

we have

$$
\begin{aligned}
& \frac{\partial^{2} L(\mathbf{p})}{\partial p_{l}^{2}}=-\frac{1}{\sigma_{\mathrm{n}}^{2}}\left\{\left(\frac{\partial^{2} \tilde{\mathbf{s}}}{\partial p_{l}^{2}}\right)^{T}\left(\mathbf{E}^{T} \mathbf{E} \tilde{\mathbf{s}}-\overline{\boldsymbol{\tau}}\right)+\left(\frac{\partial \tilde{\mathbf{s}}}{\partial p_{l}}\right)^{T} \mathbf{E}^{T} \mathbf{E} \frac{\partial \tilde{\mathbf{s}}}{\partial p_{l}}\right\} \\
& \frac{\partial^{2} L(\mathbf{p})}{\partial p_{j} \partial p_{l}}=-\frac{1}{\sigma_{\mathrm{n}}^{2}}\left\{\left(\frac{\partial^{2} \tilde{\mathbf{s}}}{\partial p_{j} \partial p_{l}}\right)^{T}\left(\mathbf{E}^{T} \mathbf{E} \tilde{\mathbf{s}}-\overline{\boldsymbol{\tau}}\right)+\left(\frac{\partial \tilde{\mathbf{s}}}{\partial p_{j}}\right)^{T} \mathbf{E}^{T} \mathbf{E} \frac{\partial \tilde{\mathbf{s}}}{\partial p_{l}}\right\} .
\end{aligned}
$$

Notice that it can be readily shown that $\mathrm{E}\left\{\mathbf{E}^{T} \mathbf{E} \tilde{\mathbf{s}}-\overline{\boldsymbol{\tau}}\right\}=0$. Thus, we get

$$
\begin{aligned}
& \mathrm{E}\left\{\frac{\partial^{2} L(\mathbf{p})}{\partial p_{l}^{2}}\right\}=-\frac{1}{\sigma_{\mathrm{n}}^{2}}\left(\frac{\partial \tilde{\mathbf{s}}}{\partial p_{l}}\right)^{T} \mathbf{E}^{T} \mathbf{E} \frac{\partial \tilde{\mathbf{s}}}{\partial p_{l}}, \\
& \mathrm{E}\left\{\frac{\partial^{2} L(\mathbf{p})}{\partial p_{j} \partial p_{l}}\right\}=-\frac{1}{\sigma_{\mathrm{n}}^{2}}\left(\frac{\partial \tilde{\mathbf{s}}}{\partial p_{j}}\right)^{T} \mathbf{E}^{T} \mathbf{E} \frac{\partial \tilde{\mathbf{s}}}{\partial p_{l}},
\end{aligned}
$$

where

$$
\begin{aligned}
\frac{\partial \tilde{\mathbf{s}}}{\partial p_{l}} & =\left[\frac{\partial q}{\partial p_{l}}, \frac{\partial s_{1}}{\partial p_{l}}, \cdots, \frac{\partial s_{M}}{\partial p_{l}}\right]^{T} \\
& =\left[\frac{p_{l}-t_{l}}{\|\mathbf{p}-\mathbf{t}\|}, \frac{r_{1, l}-p_{l}}{\left\|\mathbf{p}-\mathbf{r}_{1}\right\|}, \cdots, \frac{r_{M, l}-p_{l}}{\left\|\mathbf{p}-\mathbf{r}_{M}\right\|}\right]^{T}
\end{aligned}
$$

Note that $r_{i, l}$ is the $l$ th element of $\mathbf{r}_{i}$. Substituting (52) and (51) into (48), $\mathbf{F}$ is obtained. This completes the derivation of the CRLB when there are no errors in receiver position.

\subsection{Derivation of $C R L B$ when receiver position is imperfectly known}

When receiver position is erroneous, let $\boldsymbol{\beta}=\left[\mathbf{p}^{T}, \tilde{\mathbf{r}}^{T}\right]^{T}$ denote the estimated parameter. Assume that the TDOA and receiver position measurements are independent. The corresponding log-likelihood function can be 
expressed as

$$
L(\boldsymbol{\beta})=-\left\{\frac{1}{2 \sigma_{\mathrm{n}}^{2}}\left[\|\tilde{\boldsymbol{\tau}}\|^{2}-2 \overline{\boldsymbol{\tau}}^{T} \tilde{\mathbf{s}}+\tilde{\mathbf{s}}^{T} \mathbf{E}^{T} \mathbf{E} \tilde{\mathbf{s}}\right]+\frac{1}{2 \sigma_{\mathrm{e}}^{2}}\|\mathbf{r}-\tilde{\mathbf{r}}\|^{2}\right\} .
$$

The Fisher information matrix is given by

$$
\mathbf{F}_{\boldsymbol{\beta}}=\left[\begin{array}{cc}
\mathbf{F}_{\mathbf{p}} & \mathbf{F}_{\mathbf{p} \tilde{\mathbf{r}}}^{T} \\
\mathbf{F}_{\mathbf{p} \tilde{\mathbf{r}}} & \mathbf{F}_{\tilde{\mathbf{r}}}
\end{array}\right]
$$

where

$$
\begin{gathered}
\mathbf{F}_{\mathbf{p}}=-\mathrm{E}\left\{\frac{\partial^{2} L(\boldsymbol{\beta})}{\partial \mathbf{p} \partial \mathbf{p}^{T}}\right\}, \\
\mathbf{F}_{\mathbf{p} \tilde{\mathbf{r}}}=-\mathrm{E}\left\{\frac{\partial^{2} L(\boldsymbol{\beta})}{\partial \mathbf{p} \partial \tilde{\mathbf{r}}^{T}}\right\}, \\
\mathbf{F}_{\tilde{\mathbf{r}}}=-\mathrm{E}\left\{\frac{\partial^{2} L(\boldsymbol{\beta})}{\partial \tilde{\mathbf{r}} \partial \tilde{\mathbf{r}}^{T}}\right\} .
\end{gathered}
$$

It is clear from Appendix 7.1 that $\mathbf{F}_{\mathbf{p}}=\mathbf{F}=\frac{1}{\sigma_{\mathrm{n}}^{2}} \frac{\partial \tilde{\mathbf{s}}^{T}}{\partial \mathbf{p}} \mathbf{E}^{T} \mathbf{E} \frac{\partial \tilde{\mathbf{s}}}{\partial \mathbf{p}^{T}}$. On the other hand, differentiation of $L(\boldsymbol{\beta})$ w.r.t. to $\tilde{\mathbf{r}}$ gives

$$
\frac{\partial L(\boldsymbol{\beta})}{\partial \tilde{\mathbf{r}}^{T}}=-\left[\frac{1}{\sigma_{\mathrm{n}}^{2}}\left(-\overline{\boldsymbol{\tau}}^{T} \frac{\partial \tilde{\mathbf{s}}}{\partial \tilde{\mathbf{r}}^{T}}+\tilde{\mathbf{s}}^{T} \mathbf{E}^{T} \mathbf{E} \frac{\partial \tilde{\mathbf{s}}}{\partial \tilde{\mathbf{r}}^{T}}\right)+\frac{1}{\sigma_{\mathrm{e}}^{2}}(\tilde{\mathbf{r}}-\mathbf{r})^{T}\right] .
$$

It follows that the derivative of $\frac{\partial L(\boldsymbol{\beta})}{\partial \tilde{\mathbf{r}}^{T}}$ with respect to $\mathbf{p}$ is given by

$$
\frac{\partial^{2} L(\boldsymbol{\beta})}{\partial \mathbf{p} \partial \tilde{\mathbf{r}}^{T}}=-\frac{1}{\sigma_{\mathrm{n}}^{2}}\left[\left(\tilde{\mathbf{s}}^{T} \mathbf{E} \mathbf{E}^{T}-\overline{\boldsymbol{\tau}}^{T}\right) \frac{\partial^{2} \widetilde{\mathbf{s}}}{\partial \mathbf{p} \partial \tilde{\mathbf{r}}^{T}}+\frac{\partial \tilde{\mathbf{s}}^{T}}{\partial \mathbf{p}} \mathbf{E}^{T} \mathbf{E} \frac{\partial \tilde{\mathbf{s}}}{\partial \tilde{\mathbf{r}}^{T}}\right] .
$$

Since $\mathrm{E}\left\{\tilde{\mathbf{s}}^{T} \mathbf{E} \mathbf{E}^{T}-\overline{\boldsymbol{\tau}}^{T}\right\}=0$, it is clear that

$$
\mathbf{F}_{\mathbf{p} \tilde{\mathbf{r}}}=-\mathrm{E}\left\{\frac{\partial^{2} L(\boldsymbol{\beta})}{\partial \mathbf{p} \partial \tilde{\mathbf{r}}^{T}}\right\}=\frac{1}{\sigma_{\mathrm{n}}^{2}} \frac{\partial \tilde{\mathbf{s}}^{T}}{\partial \mathbf{p}} \mathbf{E}^{T} \mathbf{E} \frac{\partial \tilde{\mathbf{s}}}{\partial \tilde{\mathbf{r}}^{T}},
$$

where $n \times(M+1)$ matrix $\frac{\partial \tilde{\mathbf{s}}^{T}}{\partial \tilde{\mathbf{r}}}$ is given by

$$
\frac{\partial \tilde{\mathbf{s}}^{T}}{\partial \tilde{\mathbf{r}}}=\left[\frac{\mathbf{r}-\mathbf{p}}{\|\mathbf{r}-\mathbf{p}\|}, \mathbf{0}_{n \times 1}, \cdots, \mathbf{0}_{n \times 1}\right] .
$$


Moreover, the derivative of $\frac{\partial L(\boldsymbol{\beta})}{\partial \tilde{\mathbf{r}}^{T}}$ with respect to $\tilde{\mathbf{r}}$ is given by

$$
\frac{\partial^{2} L(\boldsymbol{\beta})}{\partial \tilde{\mathbf{r}} \partial \tilde{\mathbf{r}}^{T}}=-\left\{\frac{1}{\sigma_{\mathrm{n}}^{2}}\left[\left(\tilde{\mathbf{s}}^{T} \mathbf{E E}^{T}-\overline{\boldsymbol{\tau}}^{T}\right) \frac{\partial^{2} \tilde{\mathbf{s}}}{\partial \tilde{\mathbf{r}} \partial \tilde{\mathbf{r}}^{T}}+\frac{\partial \tilde{\mathbf{s}}^{T}}{\partial \tilde{\mathbf{r}}} \mathbf{E}^{T} \mathbf{E} \frac{\partial \tilde{\mathbf{s}}}{\partial \tilde{\mathbf{r}}^{T}}\right]+\frac{1}{\sigma_{\mathrm{n}}^{2}} \frac{\partial \tilde{\mathbf{r}}^{T}}{\partial \tilde{\mathbf{r}}}\right\}
$$

which means that

$$
\mathbf{F}_{\tilde{\mathbf{r}}}=-\mathrm{E}\left\{\frac{\partial^{2} L(\boldsymbol{\beta})}{\partial \tilde{\mathbf{r}} \partial \tilde{\mathbf{r}}^{T}}\right\}=\frac{1}{\sigma_{\mathrm{n}}^{2}} \frac{\partial \tilde{\mathbf{s}}^{T}}{\partial \tilde{\mathbf{r}}} \mathbf{E}^{T} \mathbf{E} \frac{\partial \tilde{\mathbf{s}}}{\partial \tilde{\mathbf{r}}^{T}}+\frac{1}{\sigma_{\mathrm{e}}^{2}} \mathbf{I}_{n}
$$

The derivation of CRLB for the case with receiver position error is then complete.

\section{References}

[1] H. D. Griffiths and N. R. W. Long, "Television-based bistatic radar," Proc. IEE - Radar Sonar Navig., vol. 133, no. 7, pp. 649-657, Dec. 1986.

[2] V. Koch and R. Westphal, "New approach to a multistatic passive radar sensor for air/space defense," IEEE Aerosp. Electron. Syst. Mag., vol. 10, no. 11, pp. 24-32, Nov. 1995.

[3] H. D. Griffiths and C. J. Baker, "Passive coherent location radar systems. Part 1: Performance prediction," Proc. IEE - Radar Sonar Navig., vol. 152, no. 3, pp. 153-159, June 2005.

[4] C. J. Baker, H. D. Griffiths, and I. Papoutsis, "Passive coherent location radar systems. Part 2 : Waveform properties," Proc. IEE-Radar Sonar Navig., vol. 152, no. 3, pp. 160-168, June 2005.

[5] Y. Huang, J. Benesty, G. W. Elko, and R. M. Mersereati, "Real-time passive source localization: a practical linear correction least-squares approach," IEEE Trans. Speech Audio Proc., vol. 9, no. 8, pp. 943-956, Nov. 2001. 
[6] K. W. Cheung, W.-K. Ma, and H. C. So, "Accurate approximation algorithm for TOA-based maximum likelihood mobile location using semi-definite programming," in Proc. IEEE ICASSP, Montreal, Canada, May 2004.

[7] D. Li and Y. H. Hu, "Least square solutions of energy based acoustic source localization problems," in Proc. Int. Conf. Parallel Proc., pp. 443-446, 2004.

[8] Y. Zhang, M. G. Amin, and A. Fauzia, "Localization of inanimate moving targets using dual-frequency synthetic aperture radar and time-frequency analysis," in Proc. IEEE Int. Geoscience Remote Sensing Symp., Boston, MA, pp. 33-36, July 2008.

[9] K. Yang, G. Wang and Z.-Q. Luo, "Efficient convex relaxation methods for robust target localization by a sensor network using time differences of arrivals," IEEE Trans. Sig. Proc., vol. 57, no. 7, pp. 2775-2784, July 2009.

[10] E. Xu, Z. Ding, and S. Dasgupta, "Source localization in wireless sensor networks from signal time-of-arrival measurements," IEEE Trans. Sig. Proc., vol. 59, no. 6, pp. 2887-2897, June 2011.

[11] Y. Zhang, K. Yang, and M. G. Amin, "Robust target localization in moving radar platform through semi-definite relaxation," in Proc. IEEE ICASSP, Taipei, Taiwan, pp. 2209-2212, Apr. 2009.

[12] K. C. Ho, X. Lu, and L. Kovavisaruch, "An accurate algebraic solution for moving source location using TDOA and FDOA measurements in the presence of receiver location errors: analysis and solution," IEEE Trans. Sig. Proc., vol. 55, no. 2, pp. 684-696, Feb. 2007. 
[13] A. Beck, P. Stoica, and J. Li, "Exact and approximate solutions of source localization problems," IEEE Trans. Sig. Proc., vol. 56, no. 5, pp. 1770-1778, May 2008.

[14] G. Wang, Y. Li, and N. Ansari, "A semidefinite relaxation method for source localization using TDOA and FDOA measurements," IEEE Trans. Veh. Tech., vol. 62, no. 2, pp. 853-862, Feb. 2013.

[15] X. Yin, T. Pedersen, P. Blattnig, A. Jaquier, and B. H. Fleury, "A singlestage target tracking algorithm for multistatic DVB-T passive radar systems," in Proc. IEEE Digital Signal Proc. Workshop and IEEE Signal Proc. Edu. Workshop, pp. 518-523, Marco Island, FL, 2009.

[16] U. Nickel, "System considearions for passive radar with GSM illuminators," in Proc. IEEE Int. Symp. Phased Array Sys. and Techn., pp. 189-195, Waltham, MA, 2010.

[17] J. J. Zhang and A. Papandreou-Suppappola, "MIMO radar with frequency diversity," in Proc. Int. Conf. Waveform Diversity and Design, pp. 208-212, Kissimmee, FL, Feb. 2009.

[18] N. H. Lehmann, E. Fishler, A. M. Haimovich, R. S. Blum, L. J. Cimini, and R. A. Valenzuela, "Evaluation of transmit diversity in MIMO-radar direction finding," IEEE Trans. Signal Proc., vol. 55, no. 5, pp. 2215-2225, May 2007.

[19] E. Fishler, A. Haimovich, R. S. Blum, L. J. Cimini, D. Chizhik, and R. A. Valenzuela, "Spatial diversity in radar-models and detection performance," IEEE Trans. Signal Proc., vol. 54, no. 3, pp. 823-838, Mar. 2006. 
[20] C. Berger, B. Demissie, J. Heckenbach, P. Willett, and S. Zhou, "Signal processing for passive radar using OFDM waveforms," IEEE J. Selected Topics in Signal Proc., vol. 4 , no. 1, pp. 226-238, Feb. 2010.

[21] Y. D. Zhang and B. Himed, "Moving target parameter estimation and SFN ghost rejection in multistatic passive radar," in Proc. IEEE Radar Conf., Ottawa, Canada, April 2013.

[22] G. R. Curry, Radar System Performance Modeling, Artech House, 2004.

[23] Z.-Q. Luo, W.-K. Ma, A. M.-C. So, Y. Ye, and S. Zhang, "Semidefinite relaxation of quadratic optimization problems: From its practical deployments and scope of applicability to key theoretical results," IEEE Sig. Proc. Mag., vol. 27, no. 3, pp. 20-34, May 2010.

[24] S. Boyd and L. Vandenberghe, Convex Optimization, Cambridge University Press, 2004.

[25] M. S. Lobo, L. Vandenberghe, S. Boyd, and H. Lebret, "Applications of second-order cone programming," Linear Algebra and Its Applications, vol. 284, pp. 193-228, 1998.

[26] C. Cartis, N. I. M. Gould, and Ph. L. Toint, "On the complexity of steepest descent, Newton's and regularized Newton's methods for non-convex unconstrained optimization problems," SIAM J. Optim., vol. 20, no. 6, pp. 2833$2852,2009$.

[27] M. Grant and S. Boyd, CVX: Matlab Software for Disciplined Convex Programming, http://stanford.edu/ ${ }^{\text {boyd/cvx. }}$ 


\begin{tabular}{ccc}
\hline Method & Type & Complexity $(n \ll M)$ \\
\hline Proposed & Without errors, problem $(19)$ & $\tilde{\mathcal{O}}\left(n^{6.5} \bar{L}\right)$ \\
\hline SDP & Without errors $[9]$ & $\tilde{\mathcal{O}}\left(M^{6.5}\right)+\tilde{\mathcal{O}}\left(\epsilon^{-2}\right)$ \\
\hline Proposed & With errors, problem $(39)$ & $\tilde{\mathcal{O}}\left(M^{2.5} n^{2} \bar{L}\right)$ \\
\hline SDP & With errors $[9]$ & $\tilde{\mathcal{O}}\left(M^{6.5}\right)+\tilde{\mathcal{O}}\left(\epsilon^{-2}\right)$ \\
\hline
\end{tabular}

Table 1: Comparison of complexity between different methods. 


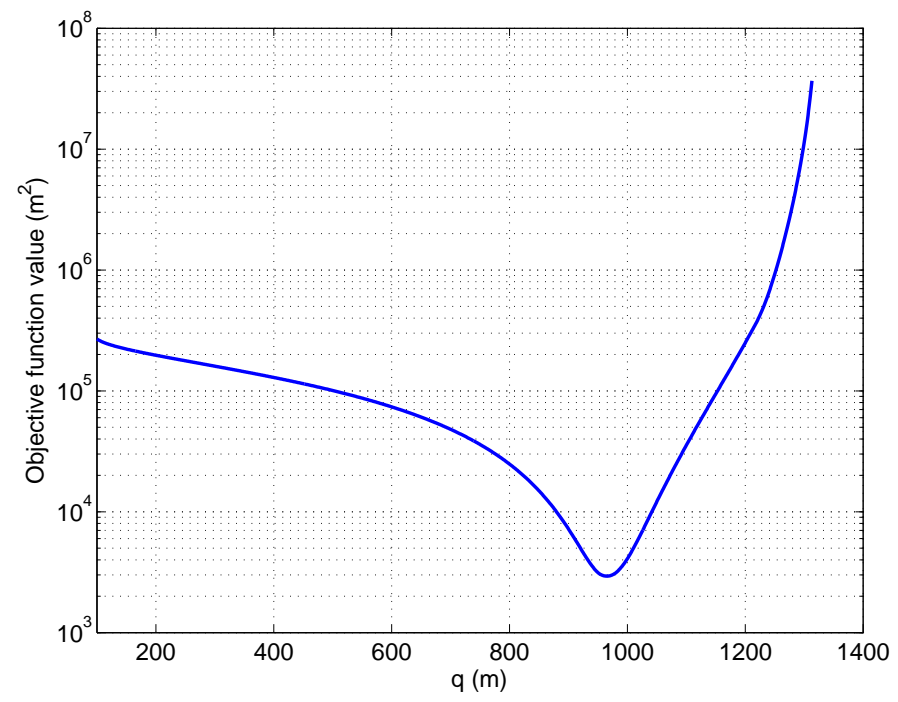

Figure 1: $\tilde{f}_{\mathrm{ob}}(q)$ versus $q$. 


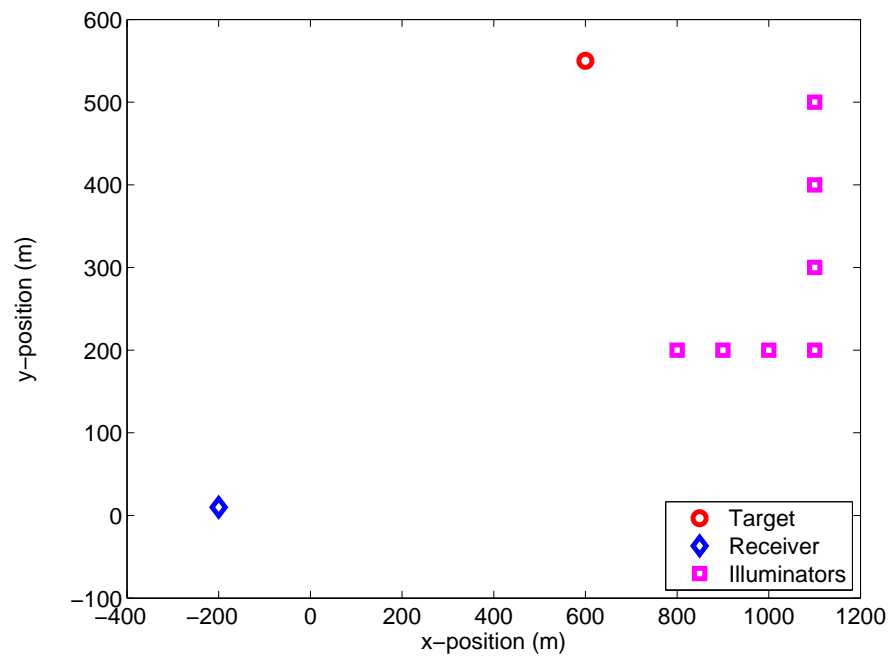

Figure 2: Scene of the simulations. 


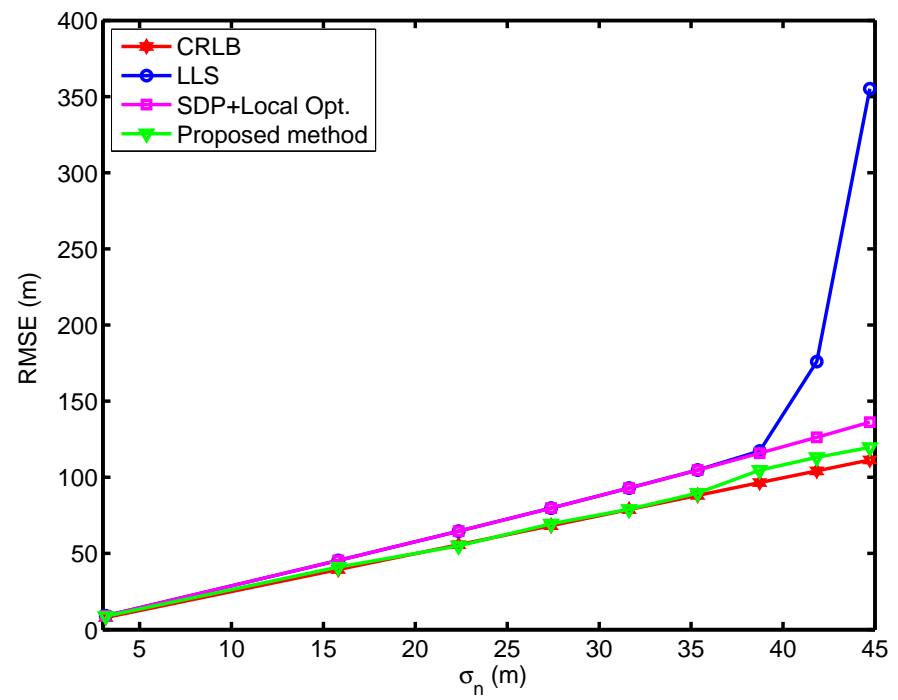

Figure 3: RMSE performance versus noise variance (no position error). 


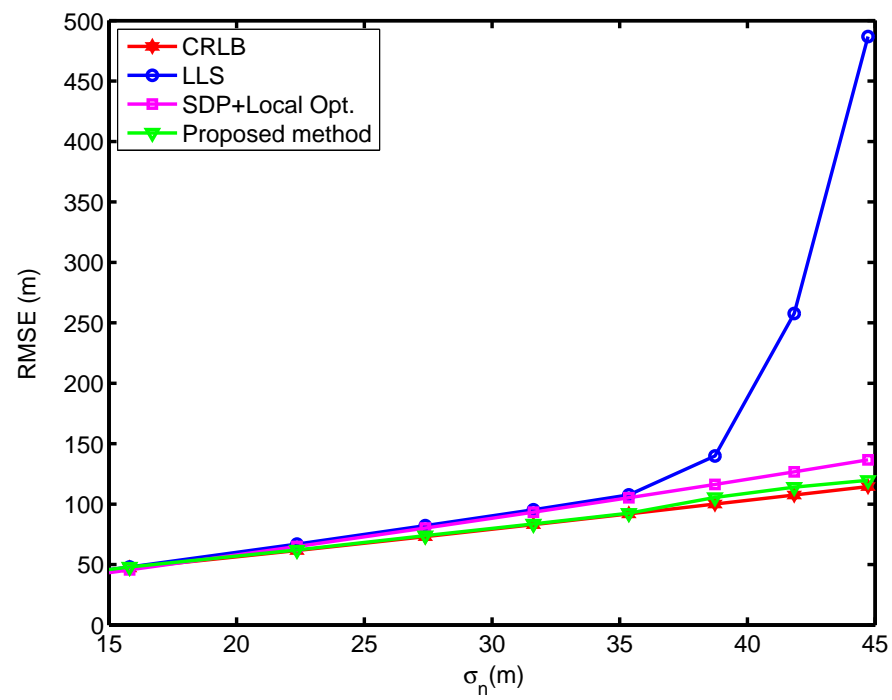

Figure 4: RMSE performance versus noise variance with uniformly distributed receiver position error $d=12 \mathrm{~m}$. 